\title{
Correction to: Anti-Sjögren's-syndrome-related antigen A autoantibodies (Anti-SSA antibody) and meningoencephalitis: Sjögren's syndrome waiting to be unveiled? A case series and review of literature
}

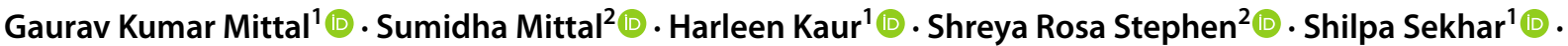 \\ Sachin Sureshbabu ${ }^{3}$ (1) Pulukool Sandhya ${ }^{2}$ P
}

Published online: 24 November 2020

(c) Springer-Verlag GmbH Germany, part of Springer Nature 2020

\section{Correction to: Rheumatology International https://doi.org/10.1007/s00296-020-04716-z}

In the original article, in the Abstract subsection, fourth line the following is stated-"To enumerate the clinical, radiological and laboratory features of meningoencephalitis related to pSS among the total cohort of meningoencephalitis admitted in our hospital." This is incorrect due to a technical mistake took place because of formatting the abstract.

The correct sentence is "This study was undertaken to enumerate the clinical, radiological and laboratory features of meningoencephalitis related to pSS among the total cohort of meningoencephalitis admitted in our hospital."

Publisher's Note Springer Nature remains neutral with regard to jurisdictional claims in published maps and institutional affiliations.

The original article can be found online at https://doi.org/10.1007/ s00296-020-04716-z.

Gaurav Kumar Mittal

drgkmittal@gmail.com

Sumidha Mittal

sumidha82@gmail.com

Harleen Kaur

harleenkaur2k9@gmail.com

Shreya Rosa Stephen

stephenshreya@gmail.com

Shilpa Sekhar

shilpasekhar.42@gmail.com

Sachin Sureshbabu

drsachins1@ rediffmail.com

Pulukool Sandhya

drsandhya.p123@gmail.com

1 Department of Neurology, St. Stephen's Hospital, Delhi 110054, India

2 Department of Rheumatology, St. Stephen's Hospital, Delhi 110054, India

3 Department of Neurology, Aster Malabar Institute of Medical Sciences, Calicut, Kerela, India 